\section{P1-21 CLUSTER RANDOMISED TRIALS IN A HEALTHCARE DATABASE: UTILISING ELECTRONIC PATIENT RECORDS FOR INTERVENTION RESEARCH}

doi:10.1136/jech.2011.142976c.15

${ }^{1} \mathrm{~A}$ Dregan, ${ }^{*} \mathrm{~L} \mathrm{~L}$ McDermott, ${ }^{3} \mathrm{G}$ McCann, ${ }^{2} \mathrm{~L}$ Yardley, ${ }^{3} \mathrm{~T}$ van Staa, ${ }^{1} \mathrm{M}$ Gulliford, ${ }^{1} \mathrm{e}$ eCRT Research Team. ${ }^{1}$ King's College London, Primary Care and Public Health Sciences, London, UK; ${ }^{2}$ University of Southampton, Southampton, UK; ${ }^{3}$ GPRD Division, Medicines and Healthcare products Regulatory Agency, London, UK

Introduction Cluster randomised trials (CRTs) may have limited power because of the correlation of individual responses within clusters and the allocation of only small numbers of clusters. This ongoing study evaluates whether a CRT may be implemented utilising the electronic patient records of the general practice research database (GPRD) that includes large numbers of UK family practices.

Methods The trial aims to reduce antibiotic prescribing for respiratory illness in primary care, randomising practices between intervention of decision support or none. The primary outcome is the proportion of consultations for respiratory illness with antibiotics prescribed. In GPRD in 2006, the proportion was approximately $39 \%$ for all RTIs; 47 practices per group will be required to detect a $5 \%$ absolute difference in this measure.

Results Research governance approvals for all primary care organisations in England and Scotland were facilitated through the Central System for Permissions. All GPRD family practices in England and Scotland were invited to participate. In the first 4 months of the study, 71 family practices were consented. Random allocation is by minimisation controlling for region and practice size. Practices allocated to the active intervention have software installed remotely to provide decision support prompts for family doctors. The intervention will continue for 12 months. Outcomes will be analysed through analysis of data for diagnosis and prescriptions recorded into GPRD.

Conclusions Healthcare databases may be used to facilitate CRTs by providing data to inform trial design, a sampling frame for recruitment, as well as data for monitoring and outcome evaluation.

\section{P1-22 WHICH MEASURES OF SOCIO-ECONOMIC POSITION PERFORM MOST CONSISTENTLY ACROSS ETHNIC GROUPS? RETROSPECTIVE COHORT STUDY USING CENSUS DATA LINKAGE}

doi:10.1136/jech.2011.142976c.16

${ }^{1} \mathrm{C}$ Fischbacher, ${ }^{2} \mathrm{G}$ Brin, ${ }^{2} \mathrm{~N}$ Bansal, ${ }^{*} \mathrm{~J}$ Pearce, ${ }^{2} \mathrm{R}$ Bhopal. ${ }^{1}$ Information Services Division, Edinburgh, UK; ${ }^{2}$ University of Edinburgh, Edinburgh, UK

Introduction Ethnic health inequalities are substantial but incompletely understood. Some studies have attributed ethnic inequalities to socio-economic status (SES) and adjusted ethnic comparisons SES measures. In the absence of individual level data, area level deprivation measures are routinely used for this purpose. However it is not clear whether these or other available SES measures perform similarly across ethnic groups. We examined the association between educational, occupational and economic indicators and incident cardiovascular disease (CVD) in ethnic groups in Scotland.

Methods We obtained educational, occupational and economic indicators for those aged over 30 years and resident in Scotland in April 2001 ( $n=2.97$ million) using the Scottish Census. Data on first CVD events up to April 2008 obtained from hospital discharge and mortality databases were combined with census sources using probability linkage methods. In each ethnic group we examined associations between CVD rates and SES indicators.
Results There were marked SES differences between ethnic groups but the SES measures were differently associated with health. In men individual educational qualification and highest household occupational group were most consistently associated across ethnic groups with CVD. Among women individual educational qualification and individual occupational group were the most consistent measures. Areal deprivation, car ownership, household tenure and employment status performed much less consistently.

Conclusions Studies of ethnic inequalities should take account of SES. Area measures of material deprivation do not predict cardiovascular outcomes consistently across ethnic groups. Educational level may provide a better means of taking account of such differences.

\section{P1-23 ANALYSIS OF CHANGING EFFECT OF CLIMATE ON HEALTH OVER LONG PERIOD OF TIME: METHODOLOGY AND APPLICATION}

doi:10.1136/jech.2011.142976c.17

${ }^{1} \mathrm{M}$ Friger, ${ }^{*}{ }^{2} \mathrm{O}$ Nadav, ${ }^{1} \mathrm{~L}$ Novack, ${ }^{2} \mathrm{G}$ Soifer. ${ }^{1}$ Ben-Gurion University of the Negev, Beer-Sheva, Israel; ${ }^{2}$ Bar-Ilan University, Ramat-Gan, Israel

Introduction Recently, analysis of health effects of climate change has become a global issue. This new and complicated problem calls for a new approach, since existing models cannot analyse changing effect of climate on health outcomes over a long period of time. We suggest methodology of analysis and estimation of changing effect of climate on health outcomes over time and apply it to estimating the effect of meteorological changes and hospitalisation and hospital mortality rates through a long time-period.

Methods Data on daily hospitalisation and hospital mortality (allcause and specific) rates for 16 years were obtained from a database of the medical center providing tertiary care to the most population of Southern Israel. Regional daily average temperature and humidity were used as meteorological variables. Three different methodologies have been developed:

1. Constructing and comparing trend functions adjusted to possible confounders of health and meteorological variables, using harmonic analysis based on generalised linear models.

2. Estimating the effect of meteorological variables on health, changing over time by using numeric derivations.

3. Constructing special spline models for analysis of significant changes in time, based on conventional meteorological health effect models.

Results The health and meteorological variables were characterised by increasing and non-linear trends. The trends of hospitalisation and age-adjusted hospital mortality rates are strongly and significantly associated with the trends of temperature and humidity.

Conclusion Increased health outcomes are significantly associated with increased heat and humidity in our region. Similar findings were obtained by three proposed methodologies.

\section{P1-24 JOINED UP DATA FOR JOINED UP THINKING-PRESCRIPTION DATA AS A SURVEILLANCE TOOL FOR DIABETES MORBIDITY}

doi:10.1136/jech.2011.142976c.18

N Gallagher, ${ }^{*}$ D O'Reilly, C Hughes. Queen's University, Belfast, UK

The introduction of a prescribing database as a new tool for disease surveillance (March 2009-March 2010), and its future potential for record linkage. The assessment of population health has traditionally focused on mortality data because it is readily available and there are systematic methods of data collection which are recognised 
internationally. However, morbidity data are now considered more relevant to current public health concerns, though inherently less accessible. We show how linkage of routine health data can supplement and complement mortality data to produce information that is pertinent to both research and policy making. We use diabetes, a worldwide chronic disease, driven by trends in obesity and ageing populations, as an exemplar. There are an estimated 285 million adults aged 20-79 living with diabetes worldwide yet there is a paucity of successful surveillance tools to measure the burden of this disease. Between March 2009 and March 2010 3.4\% of the population cashed prescriptions for anti-diabetic medication $(n=63853)$ with multilevel analysis showing an increased association with older age, males and increased deprivation. This study shows how the prevalence of diabetes in one country (Northern Ireland-c1.8 million) has been estimated from prescription data relating to treated diabetes. These estimates will be strengthened with linkages to data from laboratories measuring diabetic control and data from primary care used to inform the diabetic retinopathy screening services.

\section{P1-25 POTENTIAL BIAS IN HIV ESTIMATES USING RDS SAMPLING}

doi:10.1136/jech.2011.142976c.19

M Guimaraes, * C Machado. Federal University of Minas Gerais, Belo Horizonte, Minas Gerais, Brazil

Introduction Respondent-driven sampling (RDS) is a process used to collect data from hard-to-reach populations, such as men who have sex with men (MSM). This is a process of accessing a hidden population of interest via following links in the network of acquaintances belonging to the population, and it can be a useful epidemiological tool for estimating HIV prevalence in high risk populations. However, a typical sample arising from an RDS process on a network contains a certain degree of homophily, and in case the quantity surveyed is a given characteristic, this can generate biased results.

Methods A cross-sectional study was conducted in Belo Horizonte, Brazil, in order to estimate the HIV prevalence among MSM in 2009. Recruitment was carried out using RDS sampling. We assessed whether HIV status of the recruiter was associated with HIV status of the recruitee. Statistical analysis was carried out by McNemaŕs $\chi^{2}$.

Results We analysed 221 recruiter-recruitee pairs among the 274 MSM included in the study. The prevalence of HIV infection was $10.8 \%$ (95\% CI 4.5 to 17.8 ). The probability of recruiting an HIV positive individual was $80 \%$ when the recruiter was also HIV positive and $8.3 \%$ when the recruiter was HIV negative, indicating a dependency on HV status during the recruitment process (McNemar=13.5, $\mathrm{p}<0.001)$.

Conclusion Although RDS sampling may be suitable for ascertaining HIV prevalence among hidden populations, these estimates are likely to be biased and results should be carefully assessed and proper adjustments carried out.

\section{P1-26 THE ALL IRELAND TRAVELLER HEALTH STUDY OF TEN THOUSAND FAMILIES PROVIDES A HUMAN DEVELOPMENT INDEX FOR IRISH TRAVELLERS}

doi:10.1136/jech.2011.142976c.20

N A Hamid, ${ }^{*}$ C Kelleher, L Daly, B Quirke, P Fitzpatrick, For the All Ireland Traveller Health Study Team. University College Dublin, Belfield, Dublin 4, Ireland

Introduction Irish Travellers, a nomadic minority group in Ireland, experience multi-faceted social deprivation. The All Ireland Traveller
Health Study (AITHS) 2007-2011 of an estimated 10000 families in both Irish jurisdictions shows little improvement in most measured indicators since the last systematic assessment in 1987. The Human Development Index (HDI), measures long term basic dimensions including life expectancy, access to knowledge and an adequate standard of living. In 2010, using the improved HDI calculation, Republic of Ireland (ROI) was placed fifth (HDI=0.895) in the world league (2005-2009: 0.894). The aim of this analysis is to calculate the HDI of Irish Travellers for 2008.

Methods The AITHS census, a comprehensive 45 min census interview employing a novel audio-visual computer-assisted instrument, was conducted with Traveller families by 180 trained peer researcher teams over a 6 week period commencing on $14^{\text {th }}$ October 2008. A retrospective mortality study over past 1 year was also conducted. Data from both were used to construct the HDI for Irish Travellers.

Results The family response rate was 79\%. Life expectancy at birth was estimated at 61.7 years for men, 70.1 for women. Mean years of schooling was 8.1 (SD 4) and 94\% of Irish Travellers were below the poverty line. Calculated HDI for Irish Travellers in 2008 is 0.713 , 0.181 below ROI's HDI.

Conclusion There is a $20.2 \%$ difference in HDI for Travellers compared to the general population. This is wider than seen for other indigenous minority populations worldwide and has important cross-sectoral policy implications. (250 words).

\section{P1-27 EFFECTIVENESS OF TRAINING IN MOTIVATIONAL INTERVIEWING IN OUITTING OR REDUCING SMOKING INTENSITY IN PREGNANCY AND POST-PARTUM}

doi:10.1136/Jech.2011.142976c.21

${ }^{2} \mathrm{C}$ Hayes, ${ }^{*}{ }^{3} \mathrm{C}$ Collins, ${ }^{3} \mathrm{H}$ O'Carroll, ${ }^{3} \mathrm{E}$ Wyse, ${ }^{2} \mathrm{M}$ Gunning, ${ }^{2} \mathrm{~A}$ Rhatigan, ${ }^{3} \mathrm{M}$ Geary, ${ }^{1} \mathrm{C}$ Kelliher. ${ }^{1}$ University College Dublin, Dublin, Ireland; ${ }^{2}$ Health Service Executive, Dublin, Ireland; ${ }^{3}$ Rotunda Hospital, Dublin, Ireland

Introduction This study aimed to determine whether an integrated approach, involving training of professional staff in maternity and child health services to deliver sustained brief intervention using Motivational Interviewing (MI) techniques in a standardised way, was sufficient to affect change in smoking status or intensity in lowincome pregnant and post-partum women.

Methods 500 consecutive smokers were recruited at the first antenatal visit to receive usual care (controls). Post staff training, 500 more women were recruited (intervention group). Data were recorded at 28-32 weeks gestation and 3-4 months post-partum. The primary outcome measure was self-reported smoking cessation verified by urinary cotinine. Changes in smoking intensity were also measured.

Results There was no difference in the proportion of those who stopped smoking at $28-32$ weeks pregnancy $(8.8 \%$ vs $9.3 \%) \chi^{2}=5.9$, d.f. $=3$, $p=0.12$, or $3-4$ months post-partum $7.6 \%$ vs $7.2 \%\left(\chi^{2}=4.46\right.$, d.f. $=3, p=0.21)$. In late pregnancy cases that had stopped at baseline were more likely to stay stopped ( $26 \%$ vs $42 \%$ ). More cases who were light ( $<5$ cigs per day) or medium intensity (5-10 per day) smokers at baseline were non-smoking in late pregnancy (light, $21.3 \%$ vs $15.8 \%$, medium $6.3 \%$ vs $3.1 \%$ ). At $3-4$ months postpartum more cases smoking 5-15 cigarettes per day moved to a lower intensity $(83.7 \%$ vs $52.1 \%$ ) and fewer shifted to a higher smoking category.

Conclusion Brief intervention of the intensity offered was ineffective in affecting quit rates in late pregnancy and 3-4 months postpartum. Subtle positive changes around smoking intensity were observed. 